\title{
Readability of Character Size for Car Navigation Systems
}

\author{
Kazuhiro Fujikake ${ }^{1}$, Satoshi Hasegawa ${ }^{2}$, Masako Omori ${ }^{3}$, Hiroki Takada ${ }^{4}$, \\ and Masaru Miyao ${ }^{5}$ \\ ${ }^{1}$ Graduate School of Information Science, Nagoya University, Japan \\ fujikake@nagoya-u.jp \\ ${ }^{2}$ Department of Information Culture, Nagoya Bunri University, Japan \\ hasegawa@nagoya-bunri.ac.jp \\ ${ }^{3}$ Faculty of Home Economics, Kobe Women's University, Japan \\ masako@suma.kobe-wu.ac.jp \\ ${ }^{4}$ Gifu University of Medical Science, Japan \\ takada@u-gifu-ms.ac.jp \\ ${ }^{5}$ Information Technology Center, Nagoya University, Chikusa-ku Nagoya, Japan \\ miyao@itc.nagoya-u.ac.jp
}

\begin{abstract}
An experiment was conducted on the ease with which a car navigation system could be read by elderly, middle-aged and young people. 127 subjects (19-76 years of age) evaluated ease of reading of the displayed characters. The experiment evaluated characters displayed on a liquid crystal display (LCD) similar to those used in car navigation systems, and evaluated readability in 9 stages. Five character sizes for traffic terms and two languages (Japanese and English) were used in the experiment. The experiment was conducted with the LCD in 2 positions: directly in front of the subject and diagonal to the left (about 30 degrees). The results showed that the directly frontward position was significantly more readable than the position of diagonal to the left.
\end{abstract}

Keywords: Car navigation system, Character size, Readability.

\section{Introduction}

Use of liquid crystal displays (LCDs) in communication terminal equipment has spread rapidly, and standards for them have been established. While the sizes of LCD screens have been increased, power-saving features have also been added by reducing their size and weight. Consequently, LDCs are now used in car navigation systems. Few reports, however, have been made on the readability of characters displayed on car navigation system screens in terms of the size of the character font used or with a focus on elderly people.

In this study, the appropriate size of character fonts used in car navigation system was examined on people of all ages, including the elderly. 


\section{Experiment}

\subsection{Experiment Description}

This experiment was conducted indoors and the subjects evaluated the readability of characters displayed on LCD screen. A 9-point scale was used for the evaluation: Very easy to read (9 points), Easy to read (7), Neutral (5), Difficult to read (3), Very difficult to read (1). The subjects were told that the readability was evaluated by the legibility of the displayed characters, not by the understandability of the content. They were also instructed to tell the experimenter when the characters were too small to read.

Five character sizes for traffic terms (character height: $2 \mathrm{~mm}, 4 \mathrm{~mm}, 6 \mathrm{~mm}, 8 \mathrm{~mm}$, and $10 \mathrm{~mm}$ ) and two languages (Japanese and English) were used in the experiment. The fonts used in this experiment were Japanese MS P Gothic font in boldface type (e.g. in Japanese "Yakan-Koutu-Dome") and English Arial font (e.g. traffic jam: TRAFFIC JAM).

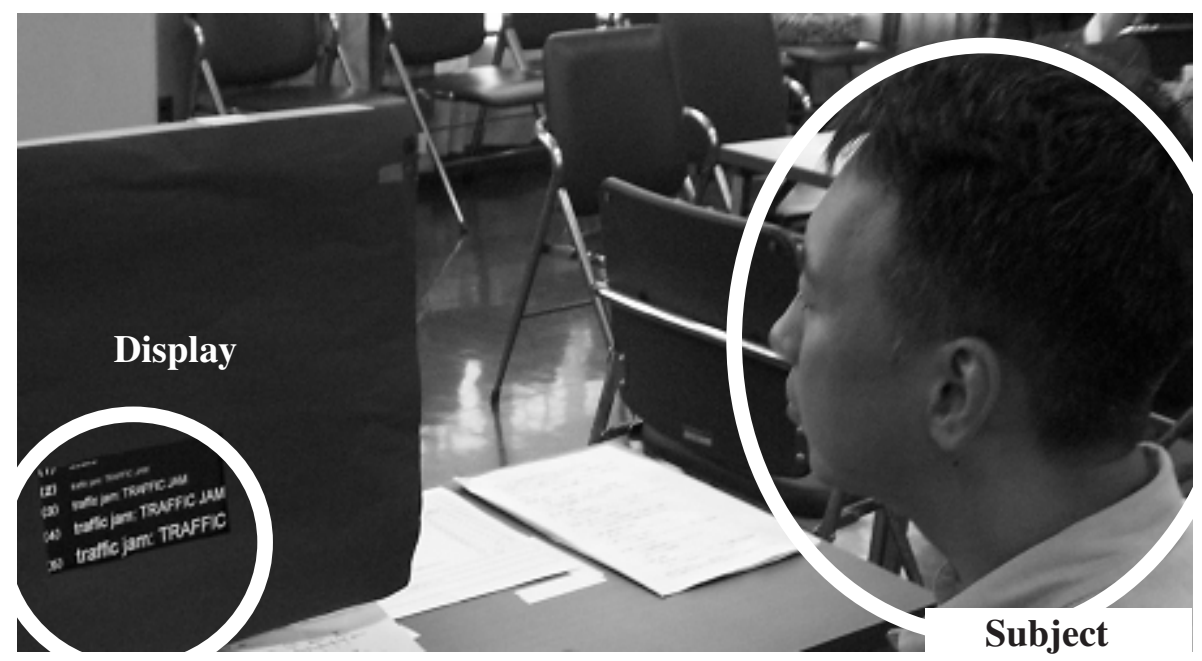

Photo. Experiment Scene

\subsection{Display Device}

A personal computer monitor (ADTEC TL700A) was used as an LCD with white characters on a black background; the ratio of luminance contrast (i.e., differences in luminance between characters and background) was 17. The illuminance was $404 \mathrm{~lx}$ in the vertical direction and $283 \mathrm{~lx}$ in the horizontal direction.

To make the LCD the same size as a car navigation system's screen, the LCD screen was covered with a black paper with an $8-\mathrm{cm}$ height/14-cm width square cutout. The characters displayed within the square cutout were evaluated as if displayed on a car navigation system's screen.

The LCD was set in 2 positions: directly in front of the subject and diagonal to the left (about 30 degrees). In both positions the LCD was set at a distance of $70 \mathrm{~cm}$ from the subjects' eyes. In addition, the subjects were told to move their head only when they looked at the screen in the left diagonal direction. 


\subsection{Subjects}

127 subjects (range 19-76 years old) with normal vision were divided into 3 groups: 72 young people (range 19-44 years old), 30 middle-aged people (range 45-64 years old), and 25 elderly people (range 65-76 years old).

The average vision at a distance of $70 \mathrm{~cm}$ was 1.2 for the young, 0.9 for the middle-aged, and 0.7 for the elderly. In addition, subjects who used eyeglasses or contact lenses in riding a bicycle on an everyday basis used them also in the experiment.

\section{Results}

The readability was evaluated for each language, LCD position, character height, and age group. Figure 1 shows the average values and standard deviations for readability of Japanese characters when the LCD was in the frontal position, for each font size and age group. Figure 2 shows the average values and standard deviations for readability of Japanese characters, with LCD in the diagonal position, for each font size and age group. Figure 3 shows the average values and standard deviations for readability of English characters, with LCD in the frontal position, for each font size and age group. Figure 4 shows the average values and standard deviations for readability of English characters, with LCD in the diagonal position, for each font size and age group.
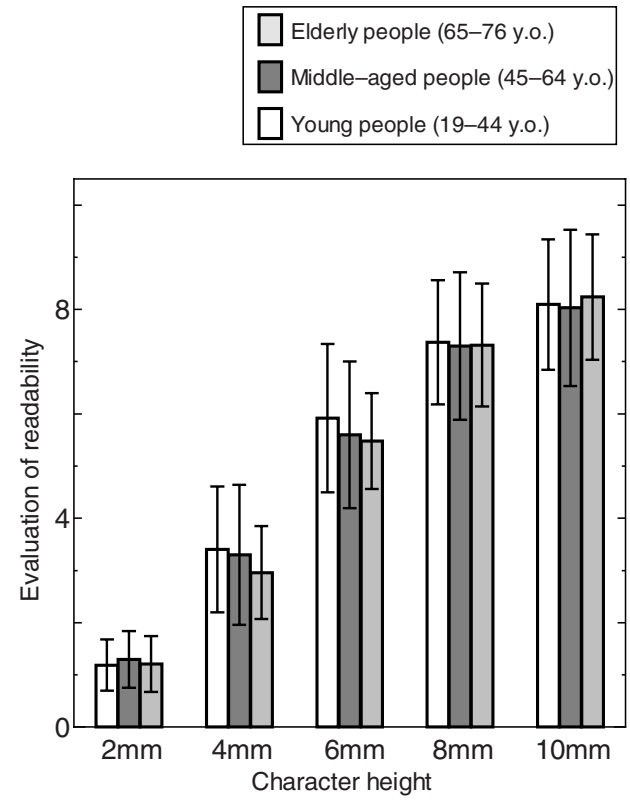

Fig. 1. Readability of Japanese characters (front view) 
$\square$ Elderly people (65-76 y.o.)

$\square$ Middle-aged people (45-64 y.o.)

$\square$ Young people (19-44 y.o.)

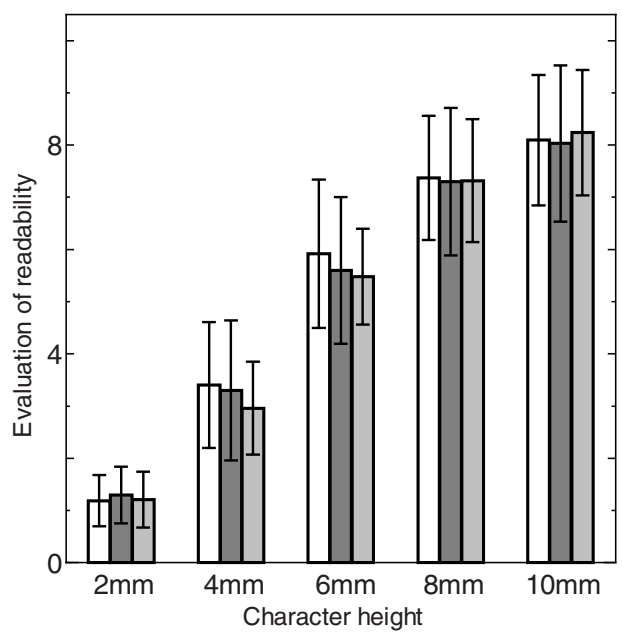

Fig. 2. Readability of Japanese characters (diagonal view)

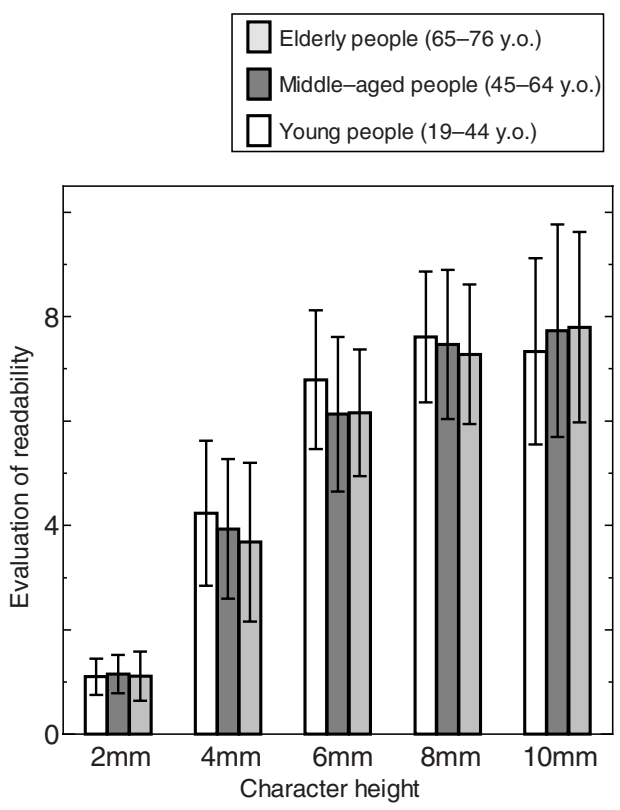

Fig. 3. Readability of English characters (front view) 


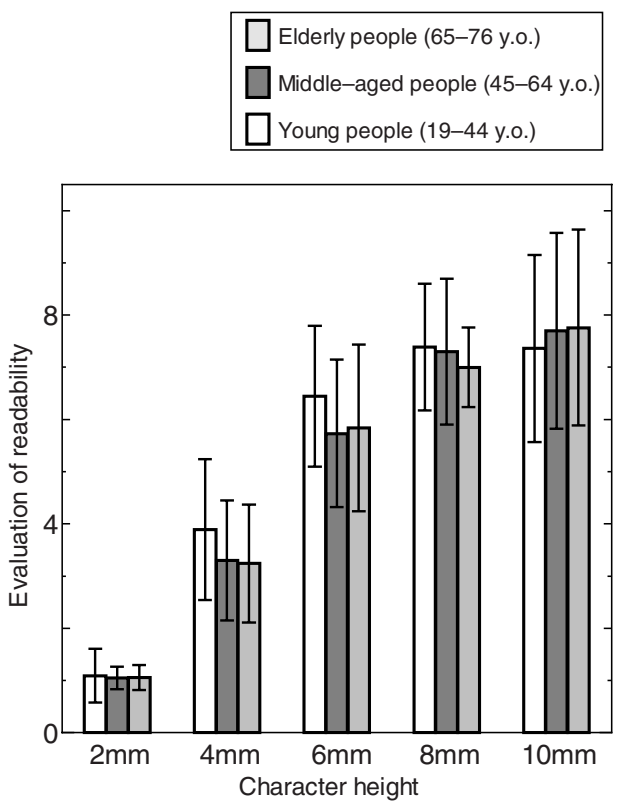

Fig. 4. Readability of English characters (diagonal view)

The overall comparison in readability between the frontal and the left diagonal positions of the LCD (paired t-test) revealed significant differences $(p<0.001)$ and it was demonstrated that readability is higher when the LCD is in the frontal position than in the left diagonal position. Readability was then evaluated according to character height (paired t-test), both in the frontal and left diagonal positions, in the two languages. Significant differences were observed $(\mathrm{p}<0.001)$ in both languages, and it was demonstrated that readability is higher in the frontal position than in the left diagonal position.

As Figure 1 shows, Japanese characters displayed in front of the subjects were the most readable at the height of $10 \mathrm{~mm}$ in all age groups and the less readable at the height of $2 \mathrm{~mm}$ in all age groups. In addition, 2 young, 3 middle-aged, and 6 elderly subjects reported that Japanese characters in the frontal direction at the height of 2 mm were too small to read.

A two-way analysis of variance was conducted for readability of Japanese characters in the frontal direction, using character heights and age groups. It revealed significant differences $(\mathrm{p}<0.001)$ between character heights, with readability increasing as character height increased. The result of a follow-up analysis of character height showed significant differences between all combinations of heights $(\mathrm{p}<0.001$ in all). In the readability of characters in the frontal direction, however, little difference was seen between the age groups.

As Figure 2 shows, Japanese characters displayed in the left diagonal were the most readable at the height of $10 \mathrm{~mm}$ in all age groups and the least readable at the height of $2 \mathrm{~mm}$ in all age groups. In addition, 3 young, 4 middle-aged, and 9 elderly subjects reported that Japanese characters in the left diagonal direction at the height of 
$2 \mathrm{~mm}$ were too small to read. Moreover, 1 middle-aged and 1 elderly subjects reported that Japanese characters in the left diagonal direction at the height of $4 \mathrm{~mm}$ were too small to read.

A two-way analysis of variance was conducted for readability of Japanese characters in the left diagonal direction, using character height and age group. It revealed significant differences $(\mathrm{p}<0.001)$ between character heights, with readability increasing as character height increased. The result of a follow-up analysis of character heights showed significant differences between all combinations of heights $(\mathrm{p}<0.001$ in all), except for the combination of $8 \mathrm{~mm}$ and $9 \mathrm{~mm}$. In addition, significant differences were seen between the age groups in the readability of characters in the left diagonal direction $(\mathrm{p}<0.01)$. The result of a follow-up analysis of age groups showed significant differences between the young and the elderly $(\mathrm{p}<0.05)$, with readability higher in the young than in the elderly.

As Figure 3 shows, English characters displayed in front of the subjects were the most readable at the height of $8 \mathrm{~mm}$ for the young and at the height of $10 \mathrm{~mm}$ for the middle-aged and the elderly. English characters were the least readable at the height of $2 \mathrm{~mm}$ in all age groups. In addition, 3 young, 4 middle-aged, and 7 elderly subjects reported that in the frontal direction English characters at the height of $2 \mathrm{~mm}$ were too small to read.

A two-way analysis of variance was conducted for readability of English characters in the frontal direction, using character heights and age groups. It revealed significant differences $(\mathrm{p}<0.001)$ between character heights, with readability increasing as character height increased. The result of a follow-up analysis of character heights showed significant differences between all combinations of heights $(\mathrm{p}<0.001$ in all), except for the combination of $8 \mathrm{~mm}$ and $9 \mathrm{~mm}$. In the readability of characters in the frontal direction, however, little difference was seen between the age groups.

As Figure 4 shows, English characters displayed in the left diagonal direction were the most readable at the height of $8 \mathrm{~mm}$ for the young and at the height of $10 \mathrm{~mm}$ for the middle-aged and the elderly. English characters were the least readable at the height of $2 \mathrm{~mm}$ in all age groups. In addition, 5 young, 9 middle-aged, and 7 elderly subjects reported that in the left diagonal direction English characters at the height of $2 \mathrm{~mm}$ were too small to read.

Finally, a two-way analysis of variance was conducted for readability of English characters in the left diagonal direction, using character heights and age groups. It showed significant differences $(\mathrm{p}<0.001)$ between character heights, with readability increased as the character height increased. The result of a follow-up analysis of character heights showed significant differences between all combinations of heights $(\mathrm{p}<0.001$ in all) except for the combination of $8 \mathrm{~mm}$ and $9 \mathrm{~mm}$.

\section{Conclusion}

Using a self-rated evaluation, display positions and character font sizes used in car navigation system were examined for readability by people of all ages, including the elderly. 
It was found that readability decreased as the font size decreased in all age groups, and in both frontal and left diagonal directions. In the readability of Japanese characters, significant differences in were found between the age groups when the display was in the left diagonal direction but not in the frontal direction. Moreover, since Japanese characters displayed in the left diagonal direction were less readable than those in the frontal direction, smaller Japanese characters in the diagonal direction were less readable for the elderly.

On the other hand, English characters were more readable than Japanese ones, one reason for which may be that English characters are simpler than Japanese ones.

\section{References}

1. International organization for standardization, Ergonomic requirements for office work with visual display terminals (VDTs) (1992) ISO 9241-3

2. International organization for standardization, Road vehicles - Ergonomic aspects of transport information and control systems - Specifications and compliance procedures for in-vehicle visual presentation (2003) ISO 15008

3. Hasegawa, S., Matsunuma, S., Omori, M., Miyao, M.: Aging effects on the visibility of graphic text on mobile phones. Gerontechnology 4(4), 200-208 (2006)

4. Hasegawa, S., Sato, K., Matsunuma, S., Miyao, M., Okamoto, K.: Multilingual disaster information system: Information delivery using graphic text for mobile phones. AI \& Society 19(3), 265-278 (2005)

5. Omori, M., Watanabe, T., Takai, J., Takada, H., Miyao, M.: Visibility and characteristics of the mobile phones for elderly people. Behavior \& Information Technology 21(5), 313-316 (2002) 